\title{
Reversible bilateral ureteric obstruction due to a pancreatic pseudocyst
}

\author{
G E Gibson, E Tiernan, C C Cronin, J B Ferriss
}

\begin{abstract}
An unusual case of bilateral ureteric obstruction and hydronephrosis due to pancreatic pseudocyst formation, after an episode of acute pancreatitis is reported. All abnormalities resolved with conservative management. Possible reasons for such ureteric obstruction include periureteric fat necrosis by pancreatic enzymes and compression by the inflammatory mass.
\end{abstract}

(Gut 1993; 34: 1267-1268)

Pancreatic pseudocysts are localised collections of pancreatic secretions occurring as a result of pancreatic inflammation and ductal disruption. ${ }^{2}$ They develop in about $10 \%$ of patients with acute alcoholic pancreatitis. Pseudocysts may be complicated by infection, haemorrhage, rupture, and by compression of adjacent organs. Bilateral ureteric obstruction due to pancreatitis has been reported once before and in that case enzymatic digestion of the ureters was the likely cause of obstruction. ${ }^{3}$ We present a case of bilateral hydroureter and hydronephrosis in which compression from a large pseudocyst seems to have been the cause. Complete resolution occurred with conservative management.

\section{Case report}

A 36 year old man with a background of heavy alcohol intake presented to another hospital with a four day history of abdominal pain, vomiting and jaundice. Plasma hepatic transaminase enzyme activities were raised and a diagnosis of alcoholic hepatitis was made. The acute symptoms resolved. Persistent pyrexia and bilateral pleuritic chest pain and effusions, however, prompted transfer to this hospital.

On admission here, he had a low grade pyrexia, bilateral pleural effusions, and moderate smooth hepatomegaly. Results of investigations included: haemoglobin $10 \mathrm{~g} / \mathrm{dl}$ (normal range (NR), 14-18 g/dl), white cell count $12.9 \times 10^{9} / 1\left(\mathrm{NR}, 4.8-10.8 \times 10^{9} / 1\right)$, platelets $673 \times 10^{9} / 1\left(\mathrm{NR}, 150-400 \times 10^{9} / 1\right)$, and an erythrocyte sedimentation rate $132 \mathrm{~mm} / \mathrm{hr}$. Mean corpuscular volume was $99 \mathrm{fl}(\mathrm{NR}, 80-92$ fl) and plasma gamma glutamyl transferase was $2.9 \mathrm{uka} / \mathrm{l}(\mathrm{NR}, 0 \cdot 1-0.8 \mathrm{uka} / \mathrm{l})$. Serum creatinine, amylase, serum aspartate transaminase, and serum alanine transaminase were within the normal range. Plasma albumin was $28 \mathrm{~g} / \mathrm{l}$ (NR, 36-44 g/l). Chest radiography confirmed bilateral pleural effusions, more considerable on the left, and linear atelectasis at the lung bases. Lung perfusion scan was normal. Pleural aspirate was an exudate.

Ultrasonography showed hydronephrosis of the left kidney, which was confirmed by intravenous urography. Computed tomography (CT) of the abdomen and pelvis showed appreciable pancreatic enlargement and widespread increase in the amount of soft tissue in the retroperitoneum extending into the pelvis, with additional soft tissue masses in the presacral region and the left side of the pelvis. The findings were highly suggestive of recent pancreatitis with pseudocyst formation.

The patient was managed conservatively with regular monitoring of renal function and of pseudocyst size. Six weeks later, CT scanning showed a bulky pancreas and a large pseudocyst of the lesser sac, with fluid tracked down on the left side of the retroperitoneum in the anterior compartment, anterior to Gerota's fascia as far as the left iliac fossa. There was now bilateral hydronephrosis with obstruction at the pelvic inlet (Figs 1 and 2). No surgical intervention was undertaken. The patient was regularly reviewed. Ultrasonography four months later showed improvement, with resolution of hydronephrosis on the left and minimal hydronephrosis on the right. CT scan, ten months after his initial presentation, showed persistent irregular enlargement of the body and the tail of pancreas with multiple small pseudocysts. Hydronephro- 


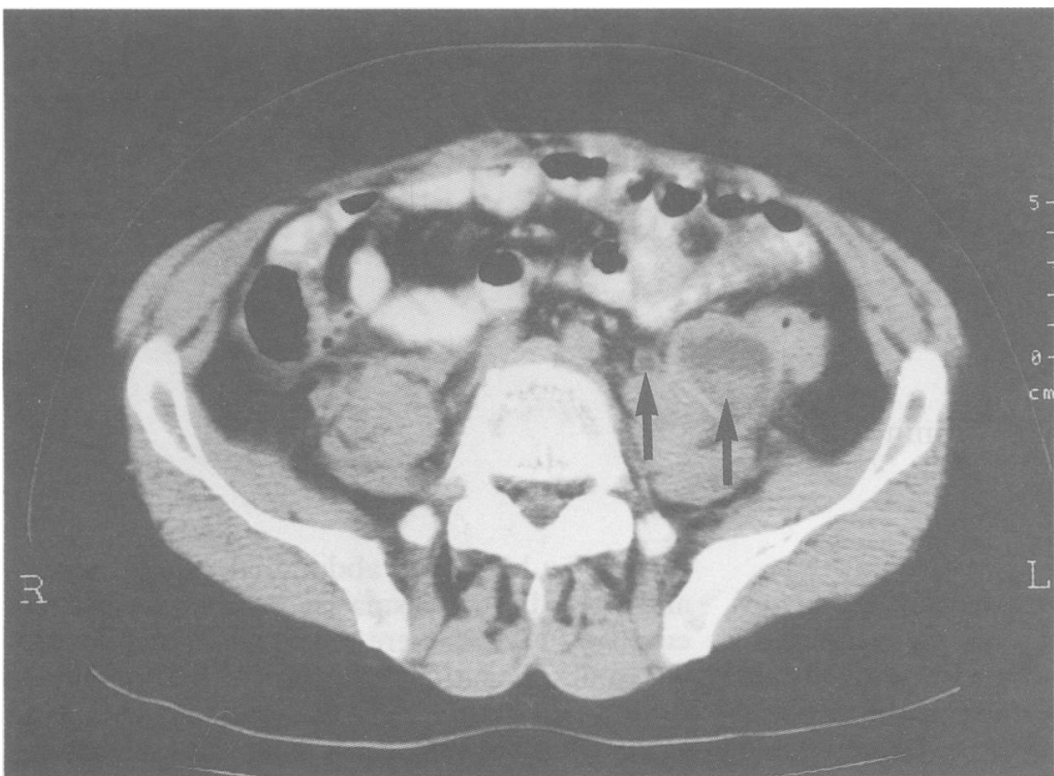

Figure 2: Computed tomography scan at the fifth lumbar vertebra showing left sided retroperitoneal mass (right arrow) abutting on ureter (left arrow).

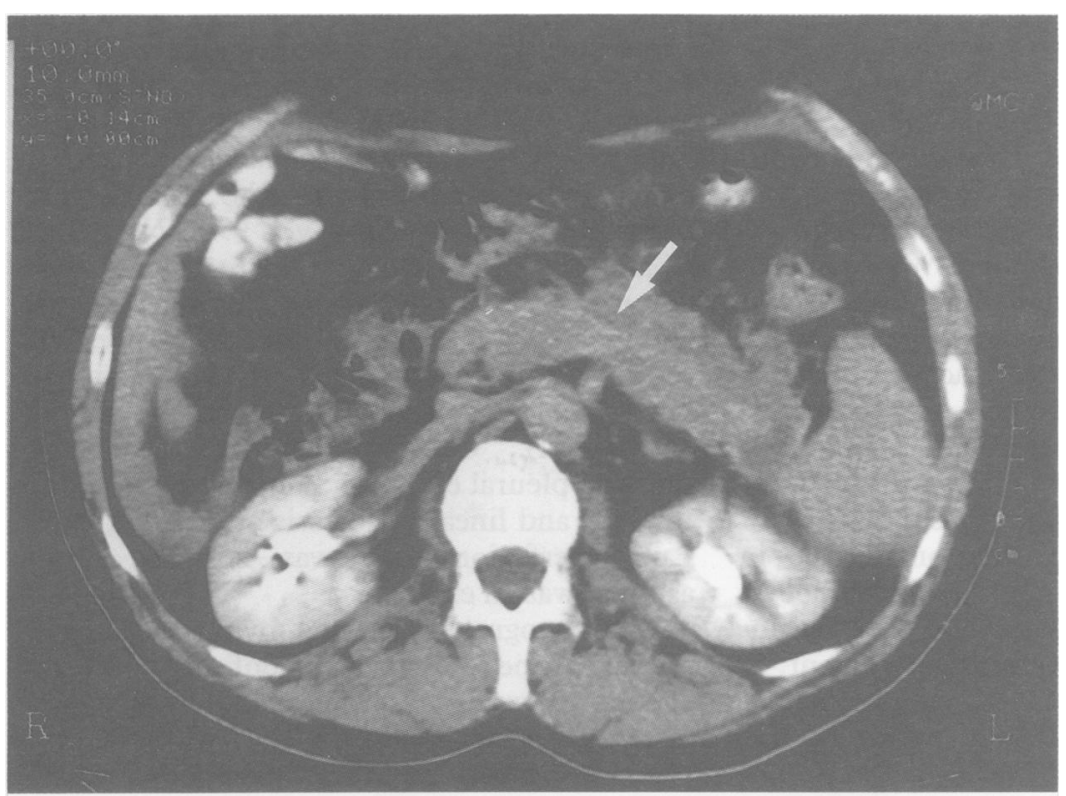

Figure 3: Computed tomography scan showing resolution of hydronephrosis. Pancreas (arrowed) remaining enlarged. sis and hydroureter had resolved (Fig 3). At all times, renal function as assessed by serum creatinine and creatinine clearance remained normal.

The patient persisted with heavy alcohol consumption and, one week following his last CT scan, died suddenly. A limited postmorten examination performed elsewhere found death due to a myocardial infarct.

\section{Discussion}

Pancreatic pseudocyst formation is a complication of severe pancreatitis and most commonly follows alcoholic pancreatitis. Pancreatic pseudocysts extend along the fascial planes, following the line of least resistance and may extend from the mediastinum ${ }^{4}$ to the groin. ${ }^{5}$ Adjacent structures may be eroded or com- pressed.$^{6}$ It is rare, however, for the urinary tract to be affected. A fistula between a pancreatic pseudocyst and the left renal pelvis has been reported, as has a left pancreatic perirenal fistula. ${ }^{6}$ Ransohoff described a patient with a pseudocyst that eroded directly into the kidney causing gross haematuria. ${ }^{7}$ Pseudocysts may compress or indent the kidney or may displace the ureter. ${ }^{8}$ Isolated ureteric obstruction is a rare complication of pancreatitis. ${ }^{9}$

One previous case of bilateral ureteric obstruction after pancreatitis has been reported and it was also in an alcoholic patient with a large pseudocyst. ${ }^{3}$ In contrast with our patient, ureteric obstruction was complete. At surgery, both ureters contained isolated necrotic segments and histological examination showed obstructed ureters with acute and chronic inflammation surrounded by fat necrosis and calcification. Direct compression of the ureters was not the cause of the obstruction, which was thought to be because of enzymatic degradation of the ureters. In contrast with our patient, although no material was available either before or after death for histological study, the absence of complete obstruction and the spontaneous resolution suggests that the ureteric obstruction was a result of compression from the pseudocyst.

There is controversy about the management of pancreatic pseudocysts. ${ }^{10}$ Operative intervention is recommended for pseudocysts greater than $6 \mathrm{~cm}$ that persist more than six weeks or that are of an indeterminate age, the rationale being the presumed high rate $(30-50 \%)$ of life threatening complications. ${ }^{11}$ Operative management is not without risk, however, as it has a reported 5-12\% death rate. Our experience with this patient agrees with the view that many such patients can be managed conservatively with careful clinical and radiological follow up.

In summary, we report a case of a retroperitoneal pancreatic pseudocyst causing distal bilateral ureteric obstruction and bilateral hydronephrosis. Complete resolution occurred with conservative management.

1 Brooks JR. Pseudocysts of the pancreas. In: Brooks JR, ed. Surgery of the pancreas. Philadelphia: Saunders, 1983: 21229.

2 Bradley III EL. Pancreatic pseudocysts. In: Bradley III EL. Complications of pancreatitis. Philadelphia: Saunders, 1982: 124-53.

3 Meller S, Stone NN, Waxman JS, Goodman A. Bilateral ureteral necrosis and obliteration secondary to pancreatic pseudocyst. F Urol 1988; 140: 1523-5.

4 Gooding GAW. Pseudocyst of the pancreas with mediastinal extension. F Clin Ultrasound 1977; 5: 121-3.

5 Salvo AF, Nematolahi $H$. Distant dissection of a pancreatic pseudocyst in the groin. Am $\mathcal{F}$ Surg 1973; 126: 430-2.

6 Sbrocchi RD, Anderson MC. Erosion of adjacent organs by pancreatic pseudocysts. Am $\mathcal{F}$ Surg 1984; 50: 85-90.

7 Ransohoff J. Pancreatitic cyst as a cause of unilateral haematuria with report of a case. Surg Gynecol Obstet 1916; 22: 275

8 Atkinson GO, Clements JL, Milledge RD, et al. Pancreatic disease simulating urinary tract disease. $\mathcal{F}$ Clin Radiol 1973; 24: 185-91.

9 Morehouse HT, Thornhill BA, Alterman DD. Right ureteral obstruction associated with pancreatitis. Urol Radiol 1985; 7: $150-2$.

10 Mullins RJ, Malangoui MA, Bergamini TM, Casey JM, Richardson JD. Controversies in the management of pancreatic pseudocysts. Am $\mathcal{F}$ Surg 1988; 155: 165-72.

11 Wade JW. Twenty-five-year experience with pancreatic pseudocysts. Am $\mathcal{F}$ Surg 1985; 149: 705-8. 